\title{
BMJ Open Correlation between symptoms and objective findings may improve the symptom-based diagnosis of chronic rhinosinusitis for primary care and epidemiological studies
}

\author{
Do-Yang Park, ${ }^{1,2}$ Eun Jung Lee, ${ }^{3}$ Ji Hoon Kim, ${ }^{3}$ Yoo Suk Kim, ${ }^{4}$ Chan Min Jung, ${ }^{3}$ \\ Kyung-Su Kim ${ }^{3}$
}

To cite: Park D-Y, Lee EJ, Kim JH, et al. Correlation between symptoms and objective findings may improve the symptom-based diagnosis of chronic rhinosinusitis for primary care and epidemiological studies. BMJ Open 2015;5: e009541. doi:10.1136/ bmjopen-2015-009541

- Prepublication history for this paper is available online. To view these files please visit the journal online (http://dx.doi.org/10.1136/ bmjopen-2015-009541).

Received 28 July 2015 Revised 17 November 2015 Accepted 18 November 2015

CrossMark

For numbered affiliations see end of article.

Correspondence to Professor Kyung-Su Kim; ydrhinol@yuhs.ac

\section{ABSTRACT}

Background: In primary care and epidemiological studies of chronic rhinosinusitis (CRS), symptombased diagnosis is common, yet limited, because endoscopic and radiological signs are not considered.

Objectives: To evaluate the correlation between symptoms and objective findings of CRS to improve its symptom-based diagnosis for primary care and epidemiological studies by using data from a large-scale nationwide epidemiological study, the Korean National Health and Nutrition Examination Survey (KNHANES).

Design: Cross-sectional study.

Setting: Data from 2008 to 2012 KNHANES.

Participants: 29225 Adults aged $>19$ years.

Outcome measures: Questionnaires targeted two major (nasal blockage and anterior/posterior nasal drip) and two minor (facial pain/pressure and reduction or loss of smell) symptoms. Nasal polyps or mucopurulent discharge from the middle meatus was defined as positive endoscopic findings for diagnosing CRS.

Results: Of the four symptoms, reduction or loss of smell was the symptom most significantly related to positive endoscopic findings in multivariable analysis (OR=1.936 (95\% Cl 1.604 to 2.337)). The combinations of symptoms showed higher ORs than individual symptoms and combinations of reduction or loss of smell with other symptoms were statistically more reliable for positive endoscopic findings than other combinations.

Conclusions: Our results show that reduction or loss of smell was the symptom most significantly related to positive nasal endoscopic findings. Therefore, symptombased diagnosis of CRS can be improved by considering reduction or loss of smell as an important symptom for positive endoscopic findings of CRS.

\section{BACKGROUND}

Chronic rhinosinusitis (CRS) is one of the most common inflammatory diseases, affecting up to $10.8 \%$ of clinic outpatients and has

\section{Strengths and limitations of this study}

- The study showed that by considering reduction or loss of smell as an important predictor of chronic rhinosinusitis (CRS), symptom-based diagnosis for primary care and epidemiological study can be improved.

- The study was conducted on a large number of population-based subjects by otorhinolaryngology experts in Korea.

- The study was based on the Korean National Health and Nutrition Examination Survey; however, in that survey, sinus CT evaluation was not conducted. Thus, this study was unable to analyse symptom correlation with CT findings for diagnosing CRS. For nasal endoscopy, nasal polyp and mucopurulent discharge from the middle meatus were regarded as positive findings; however, oedema of the middle meatus was not considered. This difference in the diagnostic criteria was related to a low incidence of positive endoscopic findings in this study.

a considerable impact on quality of life and morbidity. ${ }^{1}$ For the correct diagnosis of CRS by otolaryngological experts, the "European Position Paper on Rhinosinusitis and Nasal Polyps 2012 (EP3OS)" and the "Clinical Practice Guideline on Adult Sinusitis of the American Academy of Otolaryngology-Head and Neck Surgery (AAO-HNS) 2007" have been introduced and are used worldwide. These consensus documents contain details of symptoms, nasal endoscopic findings and radiological signs for diagnosing CRS. ${ }^{2}{ }^{3}$

These clinical guidelines incorporate all symptomatic, endoscopic and radiological criteria, in certain conditions, such as in primary care and epidemiological studies. However, the use of medical devices and resources for nasal endoscopy and 
radiological examination is impractical as it would lead to an escalation of healthcare costs. Thus, the current symptom-based diagnosis of CRS should be better designed for use in primary care and epidemiological studies. ${ }^{4}$ To improve reliability of symptom-based diagnosis, studies correlating symptoms, endoscopic findings and radiological signs have been conducted to compensate for these shortcomings. Endoscopic and radiological findings are well-correlated; however, the correlation between symptoms and endoscopic findings is inconsistent. ${ }^{5-8}$ Tomassen $e t a l^{6}$ reported the reliability of EP3OS symptom criteria and nasal endoscopy determined using data from the Global Allergy and Asthma European Network $\left(\mathrm{GA}^{2} \mathrm{LEN}\right)$ survey. In this report, $62 \%$ of enrolled subjects with positive symptoms showed positive nasal endoscopic findings and 38\% of enrolled subjects with negative symptoms showed positive nasal endoscopic findings. Thus, symptom-based CRS was statistically associated with positive endoscopy. ${ }^{6}$ If the relative value of each symptom is considered, combinations of symptoms can provide greater reliability in the diagnosis of CRS. Moreover, according to the previous study results, a large-scale epidemiological study that included endoscopic examination could facilitate correlation between symptoms and endoscopic findings and clarify this relationship. ${ }^{9}$

Recently, a large-scale nationwide epidemiological study, the Korean National Health and Nutrition Examination Survey (KNHANES), which included nasal endoscopic examination, was conducted in Korea. This study aimed to evaluate the correlation between symptoms and objective findings of CRS to improve its symptom-based diagnosis for primary care and epidemiological studies by using data from a large-scale nationwide epidemiological study, the KNHANES.

\section{MATERIAL AND METHODS}

\section{Subjects and survey methods}

The KNHANES has been conducted annually since 1998 by the Division of Chronic Disease Surveillance, Korea Centers for Disease Control and Prevention, to evaluate the health and nutritional status of Koreans. This survey uses a multistage cross-sectional, stratified sampling design without overlapping subjects. Our study was performed using data from 2008 to 2012 and included subjects aged $>19$ years.

The visiting survey team was composed of four medical experts, including an otolaryngology resident who performed the clinical evaluations in a wellequipped mobile medical examination bus. The residents had been educated and trained for standardisation of the examination by the Korean Society of Otorhinolaryngology-Head and Neck Surgery. Each participant was asked to answer a questionnaire, which included assessments of major (nasal blockage and anterior/posterior nasal drip) and minor (facial pain/ pressure and reduction or loss of smell) symptoms. After the subject had completed the questionnaire, the resident performed a physical examination. Mucopurulent rhinorrhea in the middle meatus or nasal polyps were considered positive endoscopic findings (table 1).

\section{Ethical considerations}

The survey protocol was approved by the institutional review board of the Korea Centers for Disease Control and Prevention. The participants provided written informed consent at baseline.

\section{Statistical analysis}

Univariable and multivariable logistic regression were used to analyse the association between symptoms and endoscopic findings. The relative importance of symptoms and their combinations was analysed with a logistic regression test using OR and a Forest plot. Statistical analysis was performed using SAS V.9.2 (SAS Institute Inc, Cary, North Carolina, USA). A p value $<0.05$ was considered statistically significant.

\section{RESULTS}

\section{Demographic data}

This study included 29225 subjects aged $>19$ years from a total of 38638 participants. The male-to-female ratio was $1: 1.35$ and the mean age was $50.17 \pm 16.71$ years: $50.21 \pm 16.53$ years for male and $50.14 \pm 16.85$ years for female subjects. A symptom-based diagnosis of CRS was made for 1011 subjects (3.46\% of total subjects).

Table 1 Survey items of the Korean National Health and Nutrition Examination Survey for chronic rhinosinusitis

Questions for chronic rhinosinusitis 1. Have you noted a discoloured anterior or posterior nasal drip for more than 12 weeks during the past 12 months?

2. Have you experienced nasal blockage, obstruction or congestion for more than 12 weeks during the past 12 months?

3. Have you experienced facial pain or pressure for more than 12 weeks during the past 12 months?

4. Have you experienced a reduction or loss of smell for more than 12 weeks during the past 12 months?

Endoscopic findings of chronic rhinosinusitis
1. Check if there is mucopurulent discharge from the middle meatus

2. Check if nasal polyps are present 
Association between CRS symptoms and positive endoscopic findings

The prevalence of symptoms was $0.6-5.7 \%$ (nasal drip: $4.9 \%$, nasal blockage: $5.7 \%$, facial pain: $0.6 \%$ and reduction or loss of smell: 5.2\%). Three symptoms-nasal blockage, anterior/posterior nasal drip and reduction or loss of smell-were significantly associated with the endoscopic findings on univariable logistic regression analysis (table 2). Nasal blockage and reduction or loss of smell were significantly associated with the endoscopic findings and reduction or loss of smell showed the highest OR for positive endoscopic findings in the multivariable analysis $(\mathrm{OR}=1.936$ (95\% CI 1.604 to 2.337), $\mathrm{p}<0.0001)$. However, anterior/posterior nasal drip (OR=1.217 (95\% CI 0.957 to 1.547$), \mathrm{p}=0.1085$ ) and facial pain/pressure ( $\mathrm{OR}=0.715$ ( $95 \%$ CI 0.387 to 1.321 ), $\mathrm{p}=0.2839$ ) were not associated with positive endoscopic findings in the multivariable analysis (table 2 and figure 1 ).

\section{Relative importance of symptoms and combinations of symptoms}

Four symptoms of CRS and all 11 possible combinations were analysed. The relative importance of each symptom and their combinations in comparison with nasal endoscopic findings were statistically analysed using ORs as a reference of no symptoms. The combinations of symptoms showed higher ORs than the individual symptoms. The combination of reduction or loss of smell and other symptoms showed generally higher ORs with a reliable $\mathrm{p}$ value and had a greater power of prediction for positive endoscopic findings than other combinations. The combination of nasal blockage, facial pain/pressure and reduction or loss of smell was found to have the highest OR (6.392 (95\% CI 0.845 to 48.349)). However, this combination was not statistically significant owing to its low incidence $(\mathrm{p}=0.0723)$. Thus, the combination of facial pain/pressure and reduction or loss of smell had the highest OR with statistical significance (4.154 (95\% CI 1.004 to 17.197); $\mathrm{p}=0.0494)$. In order of ORs, the combination of anterior/posterior nasal drip, nasal blockage and reduction or loss of smell (3.630 (95\% CI 2.384 to 5.529), $\mathrm{p}<0.0001)$; the combination of nasal blockage and reduction or loss of smell $(3.172$ (95\% CI 1.953 to 5.149), $\mathrm{p}<0.0001)$; and the combination of anterior/posterior nasal drip and reduction or loss of smell (2.188 (95\% CI 1.02 to 4.693$), \mathrm{p}=0.0443)$ showed high ORs (table 3 and figure 2). In the logistic regression analysis, the values for most of the combinations of facial pain/pressure and other symptoms were not estimated owing to the extreme distributions of these cases and did not show statistical significance.

\section{DISCUSSION}

\section{Synopsis of new findings}

Most primary care and epidemiological studies of CRS have an inherent weakness because large-scale endoscopic or radiological examinations are not carried out. ${ }^{10} 11$ Therefore, analysing the correlation between symptoms and endoscopic or radiological examinations of CRS might facilitate cost-effective and practical primary care and epidemiological studies. The presenting symptoms of CRS are often similar to those of other diseases and lack specificity. ${ }^{12}$ Although nasal endoscopic examinations and radiological evaluations are commonly used for diagnosis, a sinus CT scan is not cost-effective and is limited by poor specificity, demonstrating a $42 \%$ incidence of incidental mucosal abnormalities. ${ }^{13}$ Nasal endoscopic findings seem to be more objective for CRS diagnosis; however, it is often difficult for this to be performed in primary care and large-scale epidemiological studies as expensive medical devices are needed. Thus, if symptoms or their combinations are well correlated with the endoscopic findings, the acceptance of symptombased diagnosis of CRS can be improved.

In our study, a very small percentage of cases presented with facial pain/pressure or combinations including this symptom. This low incidence of facial pain/ pressure may be explained by the fact that the two major symptoms are more likely to appear at earlier

Table 2 Univariable and multivariable logistic regression analyses of association between symptoms and endoscopic findings

\begin{tabular}{|c|c|c|c|c|}
\hline \multirow[b]{2}{*}{ Symptoms } & \multicolumn{2}{|l|}{ Univariable } & \multicolumn{2}{|l|}{ Multivariable } \\
\hline & OR (95\% Cl) & p Value & OR (95\% Cl) & p Value \\
\hline \multicolumn{5}{|c|}{ Anterior/posterior nasal drip } \\
\hline Negative & 1 (reference) & & 1 (reference) & \\
\hline Positive & 1.759 (1.444 to 2.144$)$ & $<0.0001$ & 1.217 (0.957 to 1.547$)$ & 0.1085 \\
\hline \multicolumn{5}{|c|}{ Nasal blockage } \\
\hline Negative & 1 (reference) & & 1 (reference) & \\
\hline Positive & 1.950 (1.631 to 2.333 ) & $<0.0001$ & 1.563 (1.257 to 1.945$)$ & $<0.0001$ \\
\hline \multicolumn{5}{|c|}{ Facial pain/pressure } \\
\hline Negative & 1 (reference) & & 1 (reference) & \\
\hline Positive & 1.391 (0.771 to 2.509$)$ & 0.2731 & 0.715 (0.387 to 1.321$)$ & 0.2839 \\
\hline \multicolumn{5}{|c|}{ Reduction or loss of smell } \\
\hline Negative & 1 (reference) & & 1 (reference) & \\
\hline Positive & 2.197 (1.837 to 2.629$)$ & $<0.0001$ & 1.936 (1.604 to 2.337$)$ & $<0.0001$ \\
\hline
\end{tabular}


Forest plot

Odds Ratio and $95 \% \mathrm{Cl}$

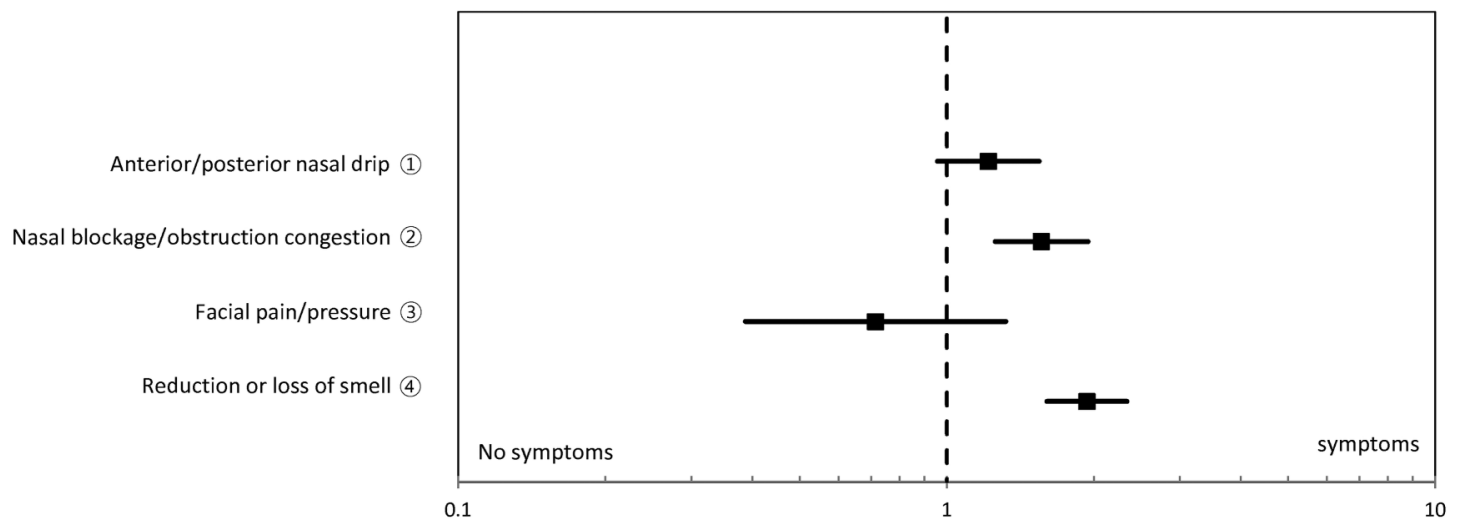

Figure 1 Forest plot of multivariate logistic regression analysis of symptoms.

stages than the minor symptoms; furthermore, minor symptoms can be confused with those of many other diseases. Another factor is the regional characteristics of medical treatment in Korea. According to the 2014 statistics of the Organization for Economic Co-operation and Development, Koreans have a high coefficient of use of medical treatment. ${ }^{14}$ Early treatment of rhinosinusitis can result in complete resolution of CRS before the development of facial pain. Thus, the incidence of facial pain/pressure and combinations including this symptom seems to be low and the values for the most of combinations of facial pain/pressure and other symptoms were not estimated owing to the extreme distributions of these cases, and did not show statistical significance.

The prevalence of CRS symptoms in this study is lower than in other reports. ${ }^{16}$ Tomassen et at ${ }^{6}$ reported the prevalence as $6.9-13.3 \%$, whereas the prevalence of symptoms in our study was $0.6-5.7 \%$. The subjects of the report by Tomassen et al were selected based on the results of a CRS questionnaire in the first cross-sectional phase by mail survey; conversely, the subjects of our study came from a general population and were selected by a multistage, cross-sectional, stratified sampling design with visiting survey. This difference in enrolment might have contributed to the difference in the prevalence. $^{15} 16$

Reduction or loss of smell showed the highest OR among the four symptoms, and the combination of reduction or loss of smell and other symptoms showed higher ORs with significantly lower $p$ values than other combinations. The combinations that best predicted endoscopic findings were those that included reduction or loss of smell, indicating that this symptom may have a greater power of prediction for positive endoscopic findings both alone and combined with other symptoms. Moreover, in our study, reduction or loss of smell, though a minor symptom of the diagnostic criteria, was found to have more predictive power than major symptoms, both

Table 3 Multiple logistic regression analyses of symptoms and their combinations compared with endoscopic findings

\begin{tabular}{|c|c|c|c|c|}
\hline \multirow[b]{2}{*}{ Symptoms } & \multicolumn{2}{|c|}{ Nasal endoscopic findings } & \multicolumn{2}{|l|}{ Logistic model } \\
\hline & Positive (\%) & Negative (\%) & OR (95\% Cl) & p Value \\
\hline No symptoms & $1166(80.41)$ & $22292(88.75)$ & 1 (reference) & \\
\hline Anterior/posterior nasal drip (1) & $35(2.41)$ & $474(1.89)$ & 1.43 (1.011 to 2.022$)$ & 0.0431 \\
\hline Nasal blockage/obstruction/congestion (2) & 52 (3.59) & 595 (2.37) & 1.685 (1.263 to 2.248$)$ & 0.0004 \\
\hline Facial pain/pressure (3) & $2(0.14)$ & $35(0.14)$ & $1.346(0.366$ to 4.943$)$ & 0.6546 \\
\hline Reduction or loss of smell (4) & $90(6.21)$ & $854(3.4)$ & 2.024 (1.617 to 2.534$)$ & $<0.0001$ \\
\hline (1) $+(2)$ & $43(2.97)$ & $432(1.72)$ & $1.922(1.399$ to 2.641$)$ & $<0.0001$ \\
\hline (1)+(3) & $1(0.07)$ & $9(0.04)$ & $3.017(0.495$ to 18.383$)$ & 0.231 \\
\hline (1) + (4) & $7(0.48)$ & $65(0.26)$ & 2.188 (1.02 to 4.693$)$ & 0.0443 \\
\hline (2) + (3) & $1(0.07)$ & $16(0.06)$ & $1.737(0.311$ to 9.711$)$ & 0.5293 \\
\hline (2)+(4) & $19(1.31)$ & $117(0.47)$ & $3.172(1.953$ to 5.149$)$ & $<0.0001$ \\
\hline (3) $+(4)$ & $2(0.14)$ & $11(0.04)$ & $4.154(1.004$ to 17.197$)$ & 0.0494 \\
\hline (1) $+(2)+(3)$ & $2(0.14)$ & $29(0.12)$ & $1.62(0.436$ to 6.02$)$ & 0.4717 \\
\hline (1) $+(2)+(4)$ & $26(1.79)$ & $139(0.55)$ & 3.63 (2.384 to 5.529$)$ & $<0.0001$ \\
\hline (1) $+(3)+(4)$ & $0(0.00)$ & $4(0.02)$ & $2.123(0.081$ to 55.711$)$ & 0.6515 \\
\hline (2) $+(3)+(4)$ & $1(0.07)$ & $4(0.02)$ & $6.392(0.845$ to 48.349$)$ & 0.0723 \\
\hline (1) $+(2)+(3)+(4)$ & $3(0.21)$ & $42(0.17)$ & $1.574(0.522$ to 4.745$)$ & 0.4206 \\
\hline
\end{tabular}


Forest plot

Odds Ratio and $95 \% \mathrm{Cl}$

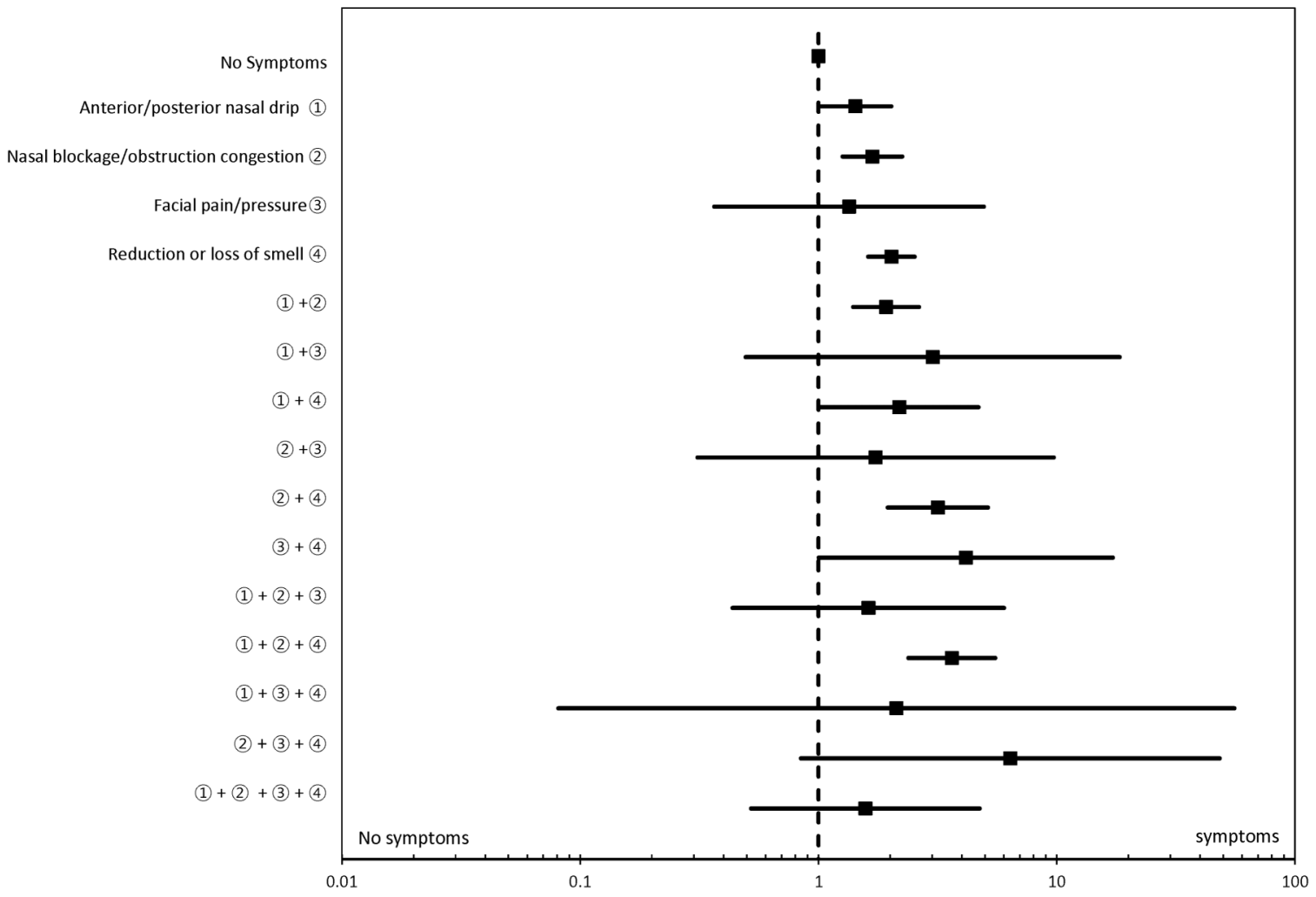

Figure 2 Forest plot of multiple logistic regression analysis of symptoms and their combinations.

individually and in combination with other symptoms. This ancillary role of reduction or loss of smell seems to be due to its aetiology. The common causes of reduction or losses of smell are upper respiratory tract infections, craniofacial trauma and unknown disease other than rhinosinusitis. ${ }^{17-19}$ Thus, this symptom is evident after other symptoms or rhinosinusitis pathologies progress. The emphasis on reduction or loss of smell has also been noted in other studies. ${ }^{9}{ }^{20}$ Pynnonen et at reported that anterior/posterior nasal drip and reduction or loss of smell are strong predictors of CRS.

\section{Limitations of the study}

Our study has some limitations. First, nasal polyps and mucopurulent discharge from the middle meatus were regarded as positive findings; however, oedema of the middle meatus was not considered, as nasal mucosal shrinkage was performed for better visualisation of the nasal cavity and reduction of pain during examination. If mucosal oedema had been included in the diagnostic criteria of CRS, the prevalence of CRS would have been greater. This difference in the diagnostic criteria in this study was related to a low incidence of positive endoscopic findings. The presence of mucosal oedema in the middle meatus will cause certain discrepancies in the prevalence of CRS since it can increase the possibility of olfactory dysfunction. Therefore, the exclusion of mucosal oedema from the diagnostic criteria is an important limitation of this study. Second, in contrast to other epidemiological studies, this study did not include sinus CT scans. This is an inevitable limitation of primary care and epidemiological studies and precluded the comparison of symptoms, endoscopic findings and/ or CT scan images. Thus, our study did not compare symptoms and symptom combinations with clinical guidelines that incorporate all symptomatic, endoscopic and radiological criteria. Further study with CT scans will be needed to more accurately determine the power of prediction. Finally, according to the 2010 Population and Housing Census in Korea, the mean age of the total population is 38.1 years old. Our study was conducted on adults aged $>19$ years, but the mean age of our patients was 50.2 years, which is somewhat high. As the reason for the prevalence of rhinosinusitis in this older age group is not clear, it is also a limitation of our study.

\section{CONCLUSION}

In primary care and epidemiological studies of CRS, symptom-based diagnosis is common yet has limitations owing to a lack of endoscopic or radiological examinations. According to our results, reduction or loss of smell was the symptom most significantly related to positive nasal endoscopic findings. Therefore, symptombased diagnosis of CRS can be improved by considering reduction or loss of smell as an important symptom for positive endoscopic findings of CRS. 
Author affiliations

${ }^{1}$ Department of Otolaryngology-Head and Neck Surgery, Soonchunhyang University College of Medicine, Cheonan Hospital, Cheonan, Republic of Korea

${ }^{2}$ Department of Medicine, Graduate School, Yonsei University, Seoul, Republic of Korea

${ }^{3}$ Department of Otorhinolaryngology, Yonsei University College of Medicine, Seoul, Republic of Korea

${ }^{4}$ Department of Otolaryngology, Ajou University School of Medicine, Suwon, Republic of Korea

Acknowledgements Hye Sun Lee, candidate and Jung Hwa Hong, biostatistician, provided statistical consultation for this study.

Contributors K-SK contributed to the conception and design of the study and to the analysis and interpretation of the study results. D-YP, EJL, JHK, YSK and $\mathrm{CMJ}$ acquired the data and conducted the statistical analyses. D-YP drafted the manuscript. All authors critically revised the manuscript and approved the final version for submission. K-SK supervised the study and is the guarantor.

Funding This research received no specific grant from any funding agency in the public, commercial or not-for-profit sectors.

Competing interest None declared.

Patient consent Obtained

Ethics approval Institutional review board of the Korea Centers for Disease Control and Prevention.

Provenance and peer review Not commissioned; externally peer reviewed.

Data sharing statement No additional data are available.

Open Access This is an Open Access article distributed in accordance with the Creative Commons Attribution Non Commercial (CC BY-NC 4.0) license, which permits others to distribute, remix, adapt, build upon this work noncommercially, and license their derivative works on different terms, provided the original work is properly cited and the use is non-commercial. See: http:// creativecommons.org/licenses/by-nc/4.0/

\section{REFERENCES}

1. Hastan D, Fokkens WJ, Bachert C, et al. Chronic rhinosinusitis in Europe-an underestimated disease. A GA ${ }^{2}$ LEN study. Allergy $2011 \cdot 66: 1216-23$

2. Fokkens WJ, Lund VJ, Mullol J, et al. European position paper on rhinosinusitis and nasal polyps 2012. Rhinol Supp/ 2012;23:3 p. Preceding table of contents, 1-298.
3. Rosenfeld RM. Clinical practice guideline on adult sinusitis Otolaryngol Head Neck Surg 2007;137:365-77.

4. Lange B, Thilsing T, Baelum J, et al. Diagnosing chronic rhinosinusitis: comparing questionnaire-based and clinical-based diagnosis. Rhinology 2013;51:128-36.

5. Kaplan BA, Kountakis SE. Role of nasal endoscopy in patients undergoing endoscopic sinus surgery. Am J Rhinol 2004;18: $161-4$

6. Tomassen P, Newson RB, Hoffmans R, et al. Reliability of EP3OS symptom criteria and nasal endoscopy in the assessment of chronic rhinosinusitis-a GA ${ }^{2}$ LEN study. Allergy 2011;66:556-61.

7. Rosbe KW, Jones KR. Usefulness of patient symptoms and nasal endoscopy in the diagnosis of chronic sinusitis. Am J Rhinol 1998;12:167-71.

8. Stankiewicz JA, Chow JM. Nasal endoscopy and the definition and diagnosis of chronic rhinosinusitis. Otolaryngol Head Neck Surg 2002;126:623-7.

9. Pynnonen M, Fowler K, Terrell JE. Clinical predictors of chronic rhinosinusitis. Am J Rhinol 2007;21:159-63.

10. Bachert C, Van Bruaene N, Toskala E, et al. Important research questions in allergy and related diseases: 3-chronic rhinosinusitis and nasal polyposis-a GALEN study. Allergy 2009; 64:520-33.

11. Benninger MS, Ferguson BJ, Hadley JA, et al. Adult chronic rhinosinusitis: definitions, diagnosis, epidemiology and pathophysiology Otolaryngol Head Neck Surg 2003;129(3 Suppl):S1-32.

12. Pynnonen MA, Terrell JE. Conditions that masquerade as chronic rhinosinusitis: a medical record review. Arch Otolaryngol Head Neck Surg 2006;132:748-51.

13. Havas TE, Motbey JA, Gullane PJ. Prevalence of incidental abnormalities on computed tomographic scans of the paranasal sinuses. Arch Otolaryngol Head Neck Surg 1988;114:856-9.

14. Organization for Economic Co-operation and Development OECD health statistics. 2013. (accessed 18 Nov 2014).

15. Siemiatycki J. A comparison of mail, telephone and home interview strategies for household health surveys. Am J Public Health 1979;69:238-45.

16. Fowler FJ Jr. Survey research methods. Sage publications, 2013.

17. Vandenhende-Szymanski C, Hochet B, Chevalier D, et al. Olfactory cleft opacity and CT score are predictive factors of smell recovery after surgery in nasal polyposis. Rhinology 2015;53: 29-34.

18. Ciofalo A, Filiaci F, Romeo R, et al. Epidemiological aspects of olfactory dysfunction. Rhinology 2006;44:78-82.

19. Mullol J, Alobid I, Marino-Sanchez F, et al. Furthering the understanding of olfaction, prevalence of loss of smell and risk factors: a population-based survey (OLFACAT study). BMJ Open 2012;2:pii:e001256.

20. Litvack JR, Fong K, Mace J, et al. Predictors of olfactory dysfunction in patients with chronic rhinosinusitis. Laryngoscope 2008;118:2225-30. 